\title{
Hallazgos imagenológicos en cálculos abandonados en cavidad abdominal después de colecistectomía laparoscópica: serie de 7 casos y revisión de la literatura
}

\section{Imaging Findings of Stones Left in the Abdominal Cavity Following Laparascopic Cholecystectomy: A Series of Seven Cases and Literature Review}

\author{
Juan Manuel Pérez¹, Arnaldo Andrés Brito Araújo², Carolina Pérez¹, Julián Forero¹, Paola Andrea Cifuentes Grillo³, \\ Elkin Yasmany Cabrera Riascos ${ }^{3}$, Akram Kadamani ${ }^{4}$
}

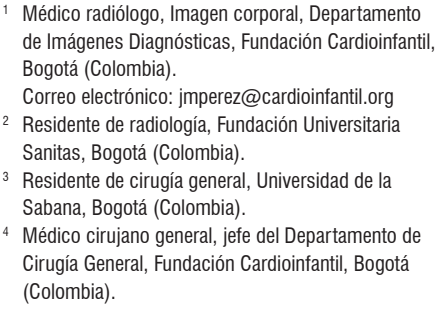
de Imágenes Diagnósticas, Fundación Cardioinfantil, Bogotá (Colombia).

Correo electrónico: jmperez@cardioinfantil.org

Residente de radiología, Fundación Universitaria

Sanitas, Bogotá (Colombia).

3 Residente de cirugía general, Universidad de la Sabana, Bogotá (Colombia).

${ }^{4}$ Médico cirujano general, jefe del Departamento de Cirugía General, Fundación Cardioinfantil, Bogotá (Colombia).

Fecha recibido: $26-09-16$ Fecha aceptado: 28-07-17

\begin{abstract}
Resumen
La colecistectomía laparoscópica $(\mathrm{CL})$ es la técnica quirúrgica de elección actual para el tratamiento de la colelitiasis sintomática. Sin embargo, este procedimiento acarrea ciertas complicaciones, dentro de las cuales destacamos la perforación de la vesícula biliar con subsiguiente caída de los cálculos y bilis a la cavidad peritoneal, que puede dar origen a la formación de abscesos abdominales tiempo después de dicho procedimiento quirúrgico.

Por tal motivo, los cálculos biliares abandonados deben reconocerse como una fuente potencial para la formación de un proceso inflamatorio intraabdominal, en especial cuando se tiene historia de perforación vesicular durante el procedimiento, o en los casos de conversión a colecistectomía abierta y caída de los cálculos a la cavidad peritoneal. Tanto los radiólogos como los cirujanos deben familiarizarse con los hallazgos imagenológicos de las diferentes modalidades diagnósticas, como el ultrasonido, la TC y la resonancia magnética, así como con las diferentes manifestaciones clínicas que pueden presentarse.

En esta publicación, presentamos los casos de 7 pacientes con antecedente de colecistectomía laparoscópica que acudieron al servicio de urgencia de nuestro hospital por dolor abdominal y en quienes los hallazgos imagenológicos determinaron la presencia de colecciones perihepáticas por cálculos caídos.
\end{abstract}

\section{Palabras clave}

Colecistectomía, colelitiasis, colecistitis, cálculo caído.

\section{INTRODUCCIÓN}

La colecistectomía laparoscópica (CL) es la técnica quirúrgica de elección actual para el tratamiento de la colelitiasis (1), la cual ha disminuido el número de complicaciones en comparación con la colecistectomía abierta, además de ofrecer una disminución en la estancia hospitalaria y en el tiempo de recuperación en el postoperatorio (2).

Las complicaciones de la colecistectomía laparoscópica son infrecuentes, aunque conllevan una alta morbilidad para los pacientes que las presentan. Una de las complicaciones más comunes es la perforación de la vesícula biliar con subsiguiente caída de los cálculos - en ocasiones infectados-y de bilis a la cavidad peritoneal, lo que puede dar lugar a la formación de abscesos, desde localización subcapsular hepática hasta extensión al tórax e, incluso, con expectoración de cálculos en algunos casos reportados en la literatura $(1,2,3,4,5)$. La incidencia de perforación de la vesícula ocurre entre el $15 \%$ y el $40 \%$ de las colecistectomías laparoscópicas $(3,6,7,8)$.

Los hallazgos radiológicos de estas complicaciones se han descrito poco en la literatura, pero hay diversos hallazgos imagenológicos que, acompañados de la clínica y teniendo en cuenta el antecedente de CL, deben alertar al radiólogo y al clínico sobre la posibilidad de que el paciente presente una reacción inflamatoria a cálculos abandonados, los cuales se comportan en estos casos como cuerpos extraños. 


\begin{abstract}
Laparascopic cholecystectomy (LC) is the current surgical technique of choice for the treatment of symptomatic cholelithiasis. However, this procedure leads to certain complications, of which we highlight gallbladder perforation with the subsequent spillage of stones and bile into the peritoneal cavity, which may give rise to the formation of abdominal abscesses some time after the surgery.

For this reason, abandoned gallstones should be recognized as a potential source of intra-abdominal inflammatory processes, especially when there is a history of gallbladder perforation during the procedure, or in cases of conversion to open cholecystectomy and spillage of gallstones into the peritoneal cavity. Both radiologists as well as surgeons should be familiar with the imaging findings of the various diagnostic modalities such as ultrasound, CT and magnetic resonance imaging, as well as the various clinical manifestations that can present.

In this publication we present 7 cases of patients with a history of laparascopic cholecystectomy who presented to our hospital's ER with abdominal pain and in whom the imaging findings showed the presence of perihepatic collections caused by spilled stones.

\section{Keywords}

Cholecystectomy, gallstone, cholecystitis, dropped gallstone.
\end{abstract}

En este artículo, presentamos una serie de 7 pacientes con antecedente de colecistectomía laparoscópica que, luego, reingresaron al servicio de urgencias de nuestra institución con cuadros de dolor abdominal y presencia de proceso inflamatorio intraabdominal.

\section{MATERIALES Y MÉTODOS}

Este estudio consiste en una serie de casos de 7 pacientes con diagnóstico de absceso subcapsular hepático por cálculos abandonados en cavidad después de colecistectomía laparoscópica que se presentaron en la Fundación Cardioinfantil entre los años 2010 y 2013. Se reportaron las fechas de dicho procedimiento y la fecha de consulta relacionada con la complicación, y se notificó el tiempo transcurrido entre la CL y la aparición de los síntomas.

Se realizó una revisión de las historias clínicas de los 7 pacientes, que incluían las evoluciones médicas y los exámenes paraclínicos, así como los estudios microbiológicos de líquido abdominal y hemocultivos. Se recopilaron todos los estudios imagenológicos realizados a cada paciente relacionados con el diagnóstico de colección intraabdominal, que incluyen ultrasonido (US), tomografía computarizada (TC) y resonancia magnética nuclear (RM).

Todos los ultrasonidos fueron realizados enfocados en la región del abdomen con los equipos GE Logiq P5 y Siemens X300; las tomografías computarizadas fueron realizadas en equipos Siemens Somaton Sensation 64 y Siemens Emotion 16. Para las resonancias magnéticas, se empleó un equipo Philips Achieva 1,5 T.

De igual forma, se revisaron las notas operatorias disponibles de los pacientes llevados a cirugía con sospecha de colección subcapsular hepática secundaria a cálculos aban- donados. Otras notas no pudieron ser revisadas porque los pacientes fueron intervenidos en otra institución.

\section{RESULTADOS}

Todos los pacientes tenían el antecedente de colecistectomía laparoscópica por colelitiasis y colecistitis descrito en la historia clínica o como hallazgo prospectivo en las imágenes diagnósticas. En dos casos, no se obtuvieron datos de la fecha de la CL. Adicionalmente, no se tuvo acceso a las descripciones quirúrgicas de las CL en ningún caso, ya que estas fueron realizadas en otros centros hospitalarios.

El rango de tiempo transcurrido desde la CL hasta la aparición de los síntomas fue de 3 meses a 7 años, con una media de 25,8 meses; solo un paciente consultó 7 años después de la CL (Tabla 1). En 6 casos, los pacientes consultaron por dolor abdominal en el hipocondrio derecho, y un paciente consultó por dolor abdominal y sensación de masa abdominal (Tabla 2). En ningún caso se encontró irritación peritoneal en el examen físico. El tiempo promedio transcurrido desde la aparición de los síntomas hasta el diagnóstico fue de 6 meses.

En los 7 casos, se hizo el diagnóstico por imágenes: 6 casos por ultrasonido, 2 por TC y 2 por RM. En dos casos, el grupo de radiología intervencionista realizó drenaje percutáneo de la colección; sin embargo, ambos pacientes volvieron a consultar por recurrencia de las colecciones. En los casos restantes, no se realizó manejo percutáneo por el antecedente de recidiva de la colección y se prefirió manejo quirúrgico como tratamiento definitivo (Tabla 3).

En las descripciones quirúrgicas de las reintervenciones, solo se encontraron cálculos dentro de las colecciones en 1 paciente, en los demás; solo se describió la presencia de colecciones con importante reacción inflamatoria. 
Tabla 1. Tiempo de aparición de síntomas posterior a colecistectomía.

\begin{tabular}{ccc}
\hline Casos & $\begin{array}{c}\text { Tiempo de aparición } \\
\text { de los síntomas (en } \\
\text { meses) }\end{array}$ & $\begin{array}{c}\text { Tiempo transcurrido entre } \\
\text { los síntomas y el diagnóstico } \\
\text { definitivo (en meses) }\end{array}$ \\
\hline Caso 1 & 3 & 1 \\
Caso 2 & - & 8 \\
Caso 3 & 18 & 18 \\
Caso 4 & 84 & 2 \\
Caso 5 & 12 & - \\
Caso 6 & 12 & 1 \\
Caso 7 & - & - \\
Promedio & 25,8 & 6 \\
\hline
\end{tabular}

Tabla 2. Sintomatología.

\begin{tabular}{lc}
\hline \multicolumn{1}{c}{ Motivo de consulta } & Cantidad de pacientes \\
\hline Dolor abdominal & 6 \\
Sensación de masa abdominal & 1 \\
Antecedente de absceso hepático & 2 \\
\hline
\end{tabular}

Tabla 3. Manejo.

\begin{tabular}{lc}
\hline \multicolumn{1}{c}{ Manejo } & Cantidad de pacientes \\
\hline Drenaje percutáneo & 2 \\
Laparoscopia diagnóstica & 2 \\
Conversión a laparotomía & 1 \\
Laparotomía exploratoria & 2 \\
Toracentesis y decorticación pulmonar & 1 \\
\hline
\end{tabular}

A todos los pacientes se les tomó muestra de la colección y esta fue enviada para tinción de Gram y cultivo. Los microorganismos aislados en el cultivo de las colecciones fueron Escherichia coli, Streptococcus viridans, Staphylococcus aureus y Klebsiella oxytoca. En el resto de casos, no se obtuvieron datos microbiológicos (Tabla 4). En un caso, el paciente desarrolló empiema torácico derecho demostrado por los hallazgos citológicos del líquido pleural, el cual se presentó como complicación subsiguiente a un intento de drenaje percutáneo de la colección subhepática. En ningún caso se evidenciaron clips de colecistectomía en la cavidad abdominal.

Tabla 4. Aislamiento bacteriológico en las colecciones

\begin{tabular}{cc}
\hline Casos & Microorganismo aislado \\
\hline Caso 1 & Escherichia coli \\
Caso 2 & Streptococcus viridans \\
Caso 3 & NC \\
Caso 4 & Staphylococcus aureus \\
Caso 5 & NC \\
Caso 6 & Klebsiella oxytoca \\
Caso 7 & NC \\
\hline
\end{tabular}

\section{DISCUSIÓN}

Desde 1992, el Instituto Nacional de Salud estandarizó la colecistectomía laparoscópica como el procedimiento quirúrgico de elección para la patología de la vesícula biliar, entre las que se destacan la colelitiasis, la colecistitis aguda, la disquinesia biliar, entre otras. Las ventajas respecto de la colecistectomía abierta son menor dolor postoperatorio, menor estancia hospitalaria y rápida recuperación. Estas características hacen del abordaje laparoscópico un procedimiento menos mórbido y más rentable económicamente (9).

La tasa de complicaciones reportada en la literatura mundial es del $4,8 \%$, con la lesión de la vía biliar como la complicación más mórbida, que alcanza una tasa del $0 \%$ al $2 \%(10,11)$. La perforación iatrogénica de la vesícula biliar durante la colecistectomía laparoscópica (CL) es relativamente frecuente, con una incidencia que oscila entre el $15 \%$ al $40 \%$; esta se asocia con caída de cálculos a la cavidad abdominal, también denominados cálculos abandonados, lo cual es más común durante la CL que durante la colecistectomía abierta. La prevalencia reportada de cálculos abandonados durante la CL es del 5\% al $7 \%$ en la literatura quirúrgica. Según Fitzgibbons y colaboradores, representan el $0,1 \%$ al $0,8 \%$ de las conversiones a laparotomía $(1,2,6,7,8,12,13)$.

La incidencia real de complicaciones mayores por cálculos intraperitoneales tras una CL es difícil de determinar debido a la escasa notificación de la perforación de la vesícula en las descripciones quirúrgicas. En una revisión reciente de 8 estudios que abarcaba 24936 colecistectomías laparoscópicas, Zehetner y colaboradores concluyeron que la incidencia de la perforación de la vesícula fue del $12 \%$ al 36\%, con una incidencia de cálculos caídos a la cavidad del 5\% al 19\%, y una frecuencia de cálculos perdidos (no recuperados) del $2 \%$. Se estima que el $8,5 \%$ de los cálculos retenidos conducirán a alguna complicación $(4,14,15)$.

Las complicaciones por cálculos intraperitoneales son poco frecuentes, aunque, en ocasiones, pueden conllevar a consecuencias graves y a una alta morbilidad. Las complicaciones más frecuentes asociadas con los cálculos abandonados tras colecistectomía laparoscópica son el absceso de la pared abdominal (18\%) e intraabdominal (44\%); en este último grupo, el subhepático y el subfrénico son los más frecuentes (Figura 1). Mucho menos frecuente son los torácicos (12\%) y los retroperitoneales (10\%). Sin embargo, el espectro de complicaciones asociado con cálculos abandonados también incluye infección de la herida quirúrgica, obstrucción o perforación intestinal, senos cutáneos, adherencias, fístulas (biliocutáneas, colocutáneas, biliocolocutáneas, bilioentéricas) e ictericia $(1,4,5,7)$ (Figura 2).

El tiempo de aparición de los síntomas subsiguiente a la caída de cálculos en la cavidad es variable y, de acuerdo con 


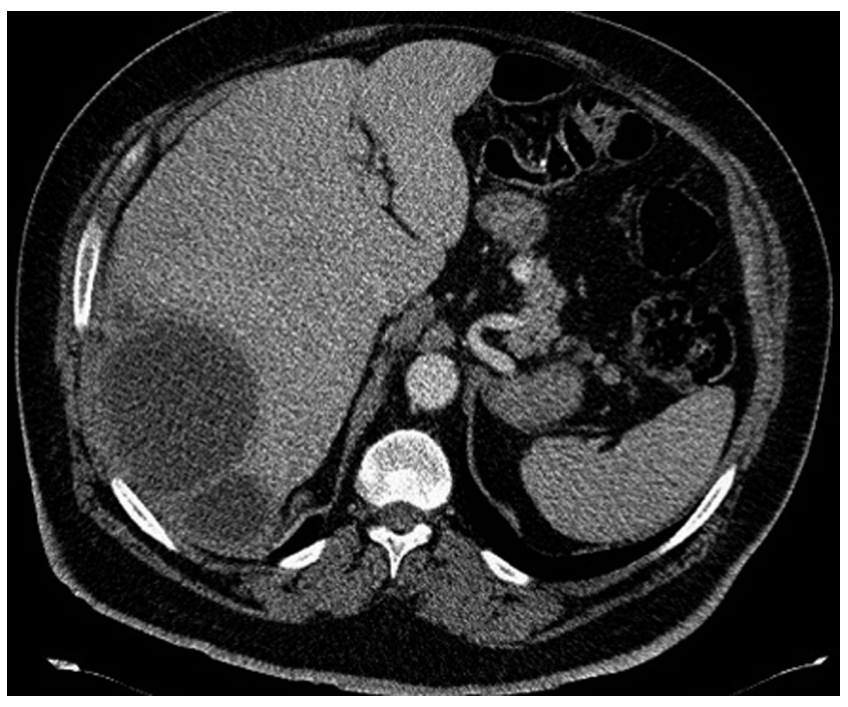

Figura 1. Tomografía computarizada contrastada de abdomen en la que se observa una colección septada subhepática posterior derecha.

series de la literatura, oscila entre pocos meses hasta años. Las manifestaciones clínicas después de los 4 años son muy infrecuentes (3). En nuestra serie, el tiempo promedio fue de 25,8 meses, y solo un caso se presentó después de 7 años. En cuanto a la fisiopatología de la formación de abscesos, se estima que los cálculos abandonados actúan como cuerpos extraños que se pueden infectar fácilmente, lo cual conduce a la formación de un proceso inflamatorio que puede llevar a la formación de abscesos en días a años luego de a la colecistectomía (16). Adicionalmente, hay diversas afecciones conocidas que se han asociado con un riesgo mayor de perforación de la vesícula durante la CL, dentro de las que se destacan la experiencia y la curva de aprendizaje del cirujano, la presencia de vesícula distendida, la colecistitis gangrenosa y una larga evolución preoperatoria del proceso.

Asimismo, Brockmann y colaboradores, en una revisión de 91 pacientes con cálculos abandonados en diferentes localizaciones, concluyeron que los factores de riesgo de desarrollar complicación secundaria a cálculos abandonados fueron colecistitis aguda con bilis infectada, caída de cálculos pigmentados, múltiples cálculos ( $>15$ cálculos), tamaño de los cálculos $(>1,5 \mathrm{~cm})$ y edad avanzada. Sin embargo, en un estudio publicado por Singh y colaboradores, no hubo diferencia estadísticamente significativa entre el tamaño y el número de cálculos con la ocurrencia de abscesos $(1,3,6,7,13,17,18)$.

Asimismo, numerosas publicaciones clínicas y experimentales han demostrado un mayor riesgo de complicaciones de los cálculos abandonados, como adherencias y colecciones intraabdominales, cuando se trata de cálculos pigmentados y en presencia de bilis infectada. Comparado con los cálculos de colesterol, los cálculos pigmentados son más pequeños y múltiples y, a menudo, conducen a inflamación y formación de adhesiones con un riesgo aumentado de perforación de la vesícula durante la CL y dificultades en la recuperación de los cálculos pigmentados caídos. Las complicaciones infecciosas son más probables que ocurran en cálculos bilirrubinados porque este tipo de cálculos contienen a menudo bacterias viables $(1,2,3,19)$.

Los microorganismos que se han cultivado y reportado en cálculos abandonados en cavidad abdominal asociados con abscesos son Escherichia coli, Klebsiella pneumoniae, Streptococcus milleri, Pseudomonas aeruginosa, Serratia marcescens, Enterobacter cloacae, Hafnea alvei o Enterococcus faecalis, microorganismos que se pueden encontrar en la

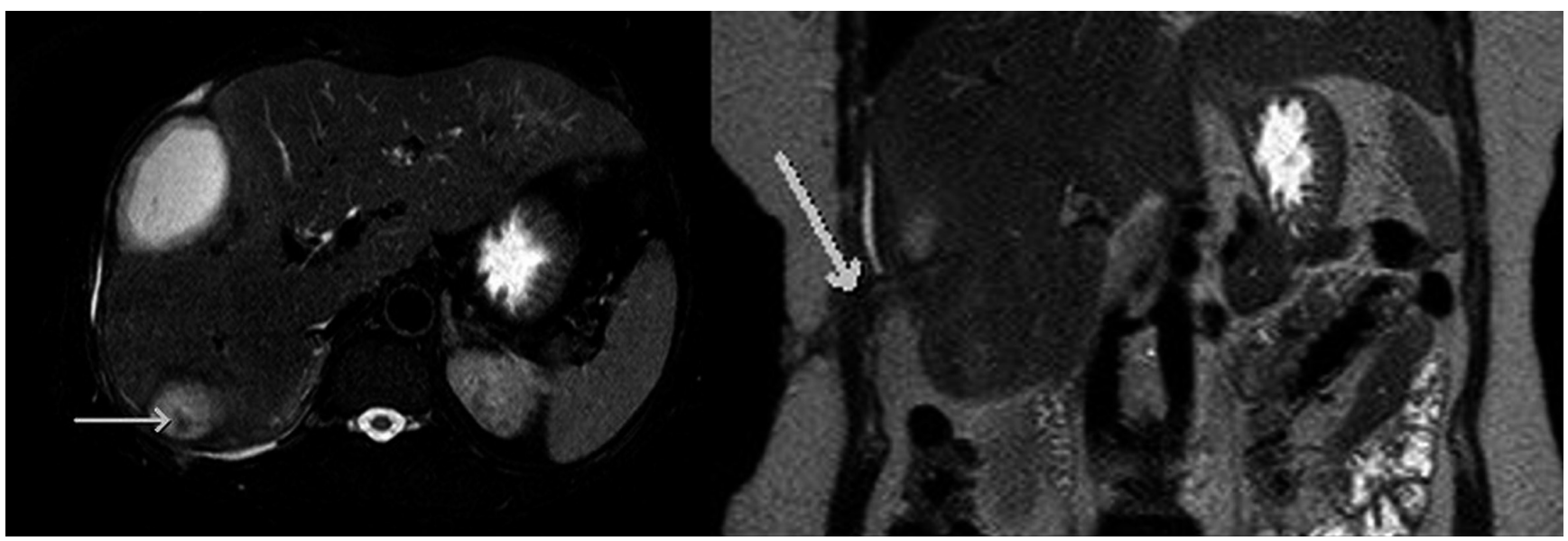

Figura 2. A. RM de abdomen con secuencia axial potenciada en T2 con supresión grasa DRIVE, en la cual se observan colecciones perihepáticas con presencia de un vacío de señal en el interior (flecha) correspondiente a un cálculo abandonado. B. Imagen coronal de RM potenciada en T2, en la cual se observa un trayecto fistuloso a la piel (flecha) en paciente con antecedente de CLP con cálculo abandonado en cavidad abdominal. 
flora normal del tracto gastrointestinal $(1,18,20,21,22)$. Contrario a los cálculos, los clips quirúrgicos caídos parecen ser un hallazgo mucho menos significativo clínicamente, ya que no están asociados con un mayor riesgo de formación de absceso y, por lo tanto, no necesitan seguimiento o remoción quirúrgica. Se han reportado pocos casos de absceso intraabdominal secundario a clips quirúrgicos de CL. Hussain reportó dos en una serie de 7 pacientes, los cuales presentaron abscesos subfrénicos recurrentes que necesitaron remoción por laparotomía. Dicho autor concluyó que no se considera su retiro de rutina debido a la naturaleza inerte y estéril de estos clips $(2,6,23,24)$ (Figura 3).

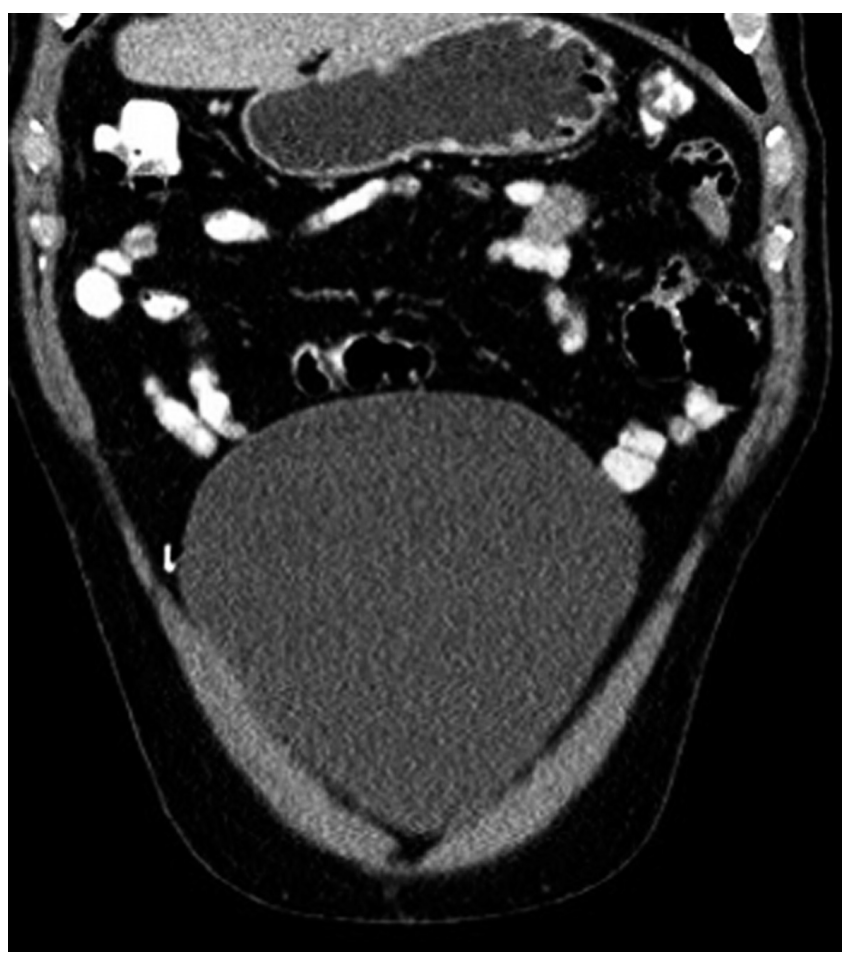

Figura 3. Reconstrucción coronal de tomografía computarizada contrastada de abdomen. Se observa un clip metálico libre después de una colecistectomía laparoscópica. Nótese la ausencia de reacción inflamatoria al rededor del clip (flecha).

En cuanto a los hallazgos imagenológicos, en la ecografía, los cálculos abandonados se manifiestan como imágenes nodulares o lineales hiperecogénicas con sombra acústica posterior (Tabla 5).

Es conocido que todos los cálculos, independientemente de la presencia de calcio, causan este artificio en el ultrasonido $(2,3,25)$ (Figura 4). Solo hay unas pocas series de casos que describen las características imagenológicas en la TC de los cálculos abandonados luego de colecistectomía, tales como lesiones heterogéneas hipodensas, con o sin calcificación central, y realce periférico luego de la adminis- tración de contraste. También se pueden presentar como colecciones multiloculadas perihepáticas, a menudo cerca del espacio de Morrison o en el retroperitoneo inferior al espacio subhepático, algunas veces con una imagen hiperdensa en su interior, la cual corresponde al cálculo $(1,6,16$, 25) (Figura 5).

Tabla 5. Hallazgos imagenológicos.

\begin{tabular}{ll}
\hline Modalidad diagnóstica & \multicolumn{1}{c}{$\begin{array}{c}\text { Hallazgos imagenológicos de cálculos } \\
\text { abandonados }\end{array}$} \\
\hline Ecografía & $\begin{array}{l}\text { Imágenes nodulares o lineales } \\
\text { hiperecogénicas con sombra acústica } \\
\text { posterior de localización perihepática. } \\
\text { Colección perihepática. }\end{array}$ \\
Tomografía contrastada & $\begin{array}{l}\text { Lesiones heterogéneas hipodensas, con } \\
\text { de abdomen } \\
\text { o sin calcificación central (hiperdensa) } \\
\text { y realce periférico posterior a la } \\
\text { administración de contraste. } \\
\text { Colecciones multiloculadas cerca del } \\
\text { espacio de Morrison o en el retroperitoneo } \\
\text { inferior al espacio subhepático, con } \\
\text { una imagen hiperdensa en su interior, } \\
\text { correspondiente al cálculo. } \\
\text { Patrones de calcificación en los cálculos } \\
\text { abandonados (periférico, central, difuso y } \\
\text { laminado). } \\
\text { Foco de baja intensidad de señal, menor de } \\
\text { 1 cm en las secuencias T1 gradiente de eco. } \\
\text { Lesiones hipointensas en las imágenes } \\
\text { potenciadas en T2 y son iso- a hipointensas } \\
\text { en las imágenes potenciadas en T1. } \\
\text { Los cálculos de colesterol muestran señal } \\
\text { elevada en T1. }\end{array}$ \\
contrastada de \\
abdomen
\end{tabular}

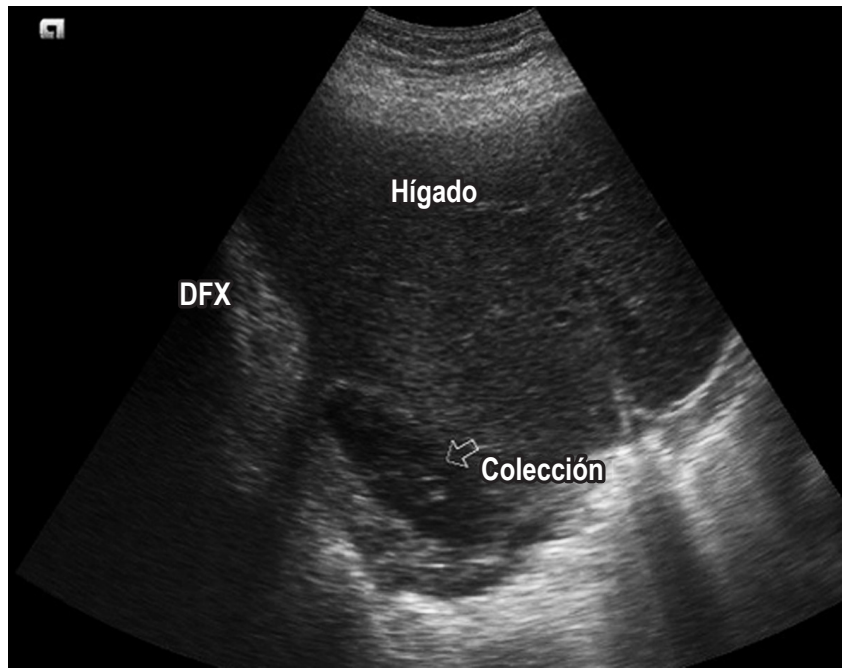

Figura 4. Imagen de una ecografía abdominal en la que se observa colección subdiafragmática secundaria a reacción a un cuerpo extraño por cálculos abandonados en cavidad. 
Los cálculos pigmentados con alto contenido de calcio son fácilmente diagnosticados con TC, mientras que los cálculos puros de colesterol y aquellos con bajo contenido de calcio pueden no ser detectados (25). Se pueden ver diferentes patrones de calcificación en los cálculos abandonados (periféricos, centrales, difusos, laminados), y pueden imitar metástasis peritoneales calcificadas (10) (Figura 6). En la resonancia magnética, se han descrito como colecciones perihepáticas multiloculadas subcapsulares, en ocasiones con compromiso de la pared abdominal que exhiben intensidad de señal heterogénea en las imágenes potenciadas en T2 y en las secuencias STIR. Una revisión subsecuente de las imágenes de MR identificaron

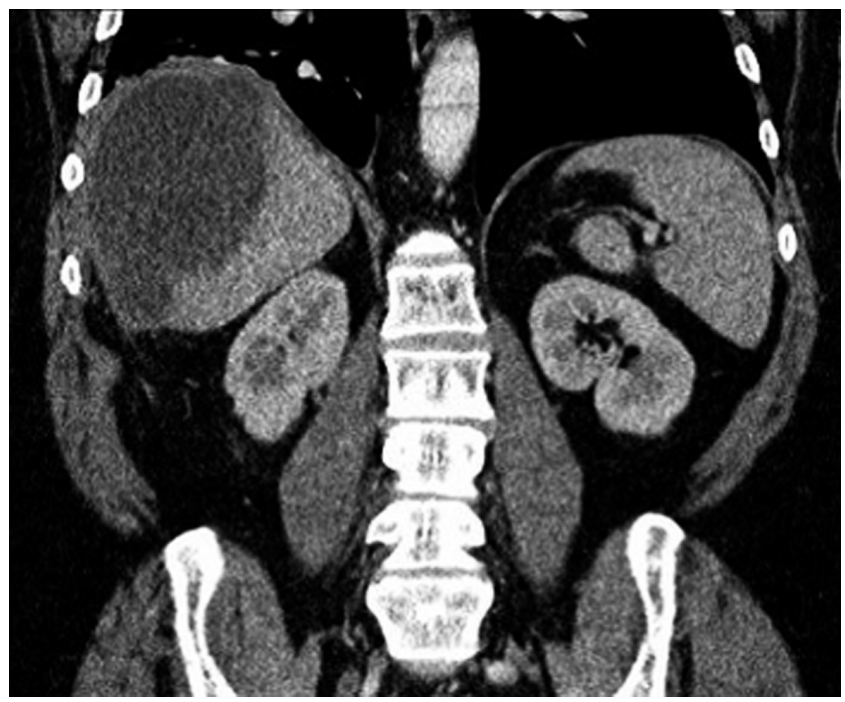

Figura 5. Reconstrucción coronal de una TC abdominal en la cual se observa colección subfrénica derecha secundaria a reacción a cálculos abandonados 4 meses después de una colecistectomía por laparoscopia en un paciente con piocolecisto. cálculos como focos de baja intensidad de señal, menor de $1 \mathrm{~cm}$ en las secuencias T1 gradiente de eco. La mayoría de los cálculos son hipointensos en las imágenes potenciadas en $\mathrm{T} 2$ y son iso a hipointensas en las imágenes potenciadas en T1, excepto en los cálculos de colesterol, los cuales pueden mostrar señal elevada en T1 $(3,10,26,27)$ (Figura 7).

Diversos reportes basados en la experiencia de expertos concluyen en que, por razones de costo y efectividad, se recomienda realizar ultrasonografía o TC antes que MR en pacientes que desarrollen colección luego de una CL (3). El diagnóstico de los abscesos intraabdominales por cálculos puede confundirse con abscesos simples y actinomicosis hepática. También se ha encontrado que los abs-

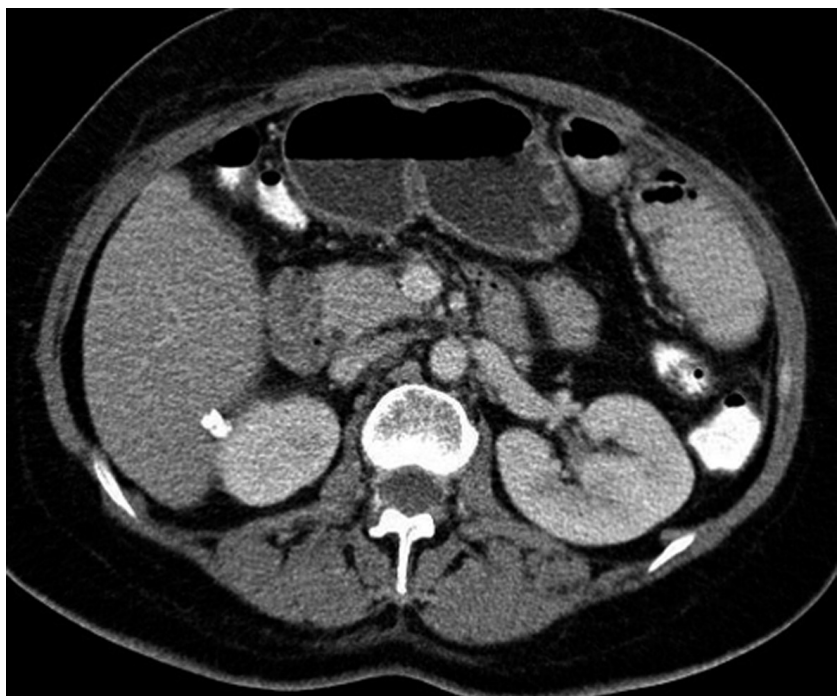

Figura 6. TC abdominal contrastada en corte axial. Se observan calcificaciones en espacio hepatorrenal, las cuales corresponden a cálculos abandonados después de una colecistectomía por laparoscopia sin reacción a un cuerpo extraño.

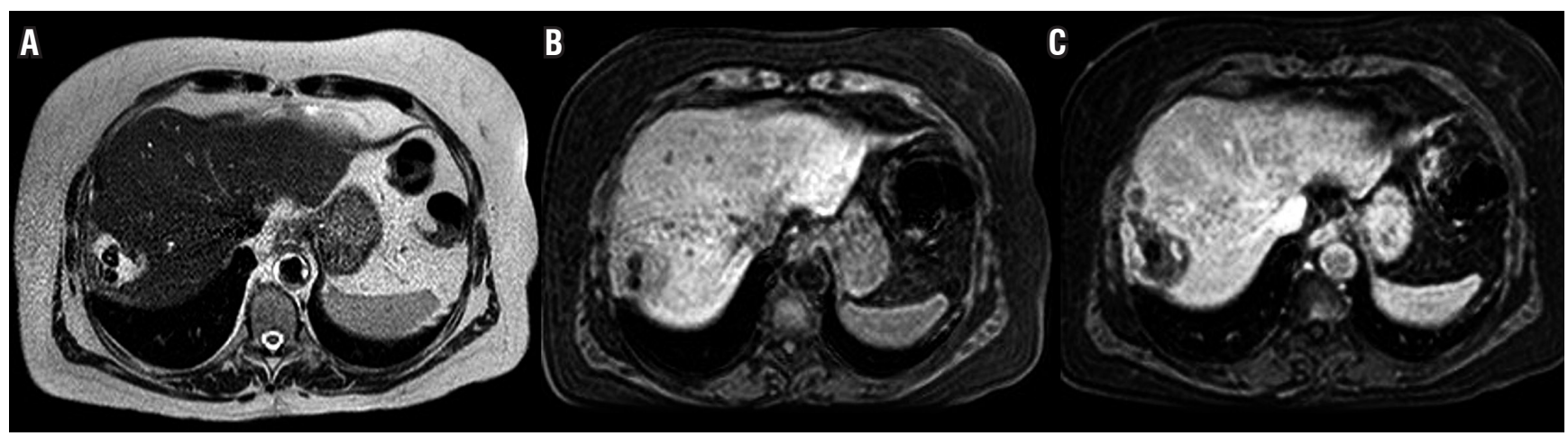

Figura 7. A. RM potenciada en T2 axial. Se identifica colección subhepática con 2 defectos hipointensos en su interior $\left({ }^{*}\right)$, los cuales corresponden a cálculos abandonados. B. RM potenciada en T1 axial. Se identifica colección subhepática con 2 defectos hipointensos en su interior $\left(^{*}\right)$, los cuales corresponden a cálculos abandonados. C. Imagen axial de RM contrastada en secuencia T1 en la cual se observa realce periférico de la colección perihepática derecha secundaria a cálculos abandonados. 
cesos por cálculos abandonados pueden imitar procesos neoplásicos, enfermedad metastásica peritoneal o procesos como la peritonitis esclerosante, que, a veces, conduce a un retraso en el diagnóstico y el tratamiento; sin embargo, el antecedente de colecistectomía por laparoscopia y la presencia de calcificación favorece el diagnóstico de cálculo abandonado $(1-3,6,13,16)$ (Figura 8).

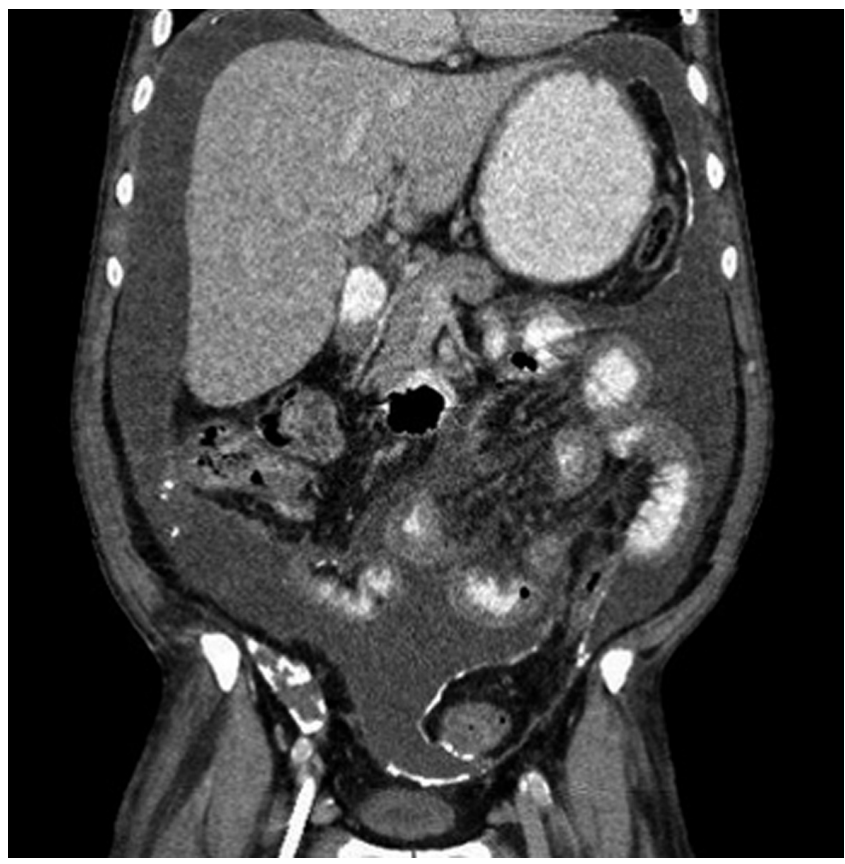

Figura 8. Reconstrucción coronal de tomografía computarizada de abdomen en un paciente con diagnóstico de peritonitis retráctil por diálisis. Nótese la presencia de múltiples calcificaciones irregulares sobre los repliegues peritoneales, las cuales no deben confundirse con litos.

Es contradictorio que no exista un consenso para el manejo de los cálculos abandonados en cavidad abdominal. Esto se explica porque algunos cirujanos consideran que la tasa de complicaciones es menor de la morbilidad que implica la conversión del procedimiento a laparotomía para extracción de los cálculos, cuando técnicamente no es posible extraerlos por laparoscopia. Soper y colaboradores analizaron 10174 colecistectomías laparoscópicas en las que evidenciaron que la mortalidad y la incidencia de complicaciones serias es significativamente menor que la conversión a laparotomía para extracción de los litos (28). Tumer y colaboradores realizaron un estudio prospectivo donde incluyeron 1528 pacientes llevados a colecistectomía laparoscópica, entre 1992 y 2002. La perforación de la vesícula biliar y el abandono de cálculos en cavidad abdominal se dio en el 3,8\% de los casos. Se documentaron cálculos abandonados en 49 pacientes, y 7 de estos presentaron complicaciones relacionadas con este evento. Dos de estos pacientes presentaron dolor o íleo intestinal que requirió manejo médico, y a 5 se les diagnosticaron abscesos intraabdominales que fueron reintervenidos o drenados de manera percutánea y el cuadro se resolvió completamente (29).

Rice y colaboradores realizaron un estudio prospectivo en el que incluyeron a 1059 pacientes con colecistectomía laparoscópica, en el que demostraron que la perforación vesicular con contaminación de la cavidad abdominal por bilis y cálculos se asocia con una mayor incidencia de abscesos intraabdominales (30). El tratamiento de las complicaciones relacionadas por cálculos biliares abandonados depende, entonces, del tipo de complicación y síntomas que estos generen. Sin embargo, en el $90 \%$ de los casos, es necesario un procedimiento quirúrgico para el drenaje del absceso intraabdominal y la extracción de los cálculos, debido a que, si no se retiran los cálculos abandonados, el absceso recurrirá y aumentará la movilidad del paciente $(16,25)$ (Figura 9). La tasa de reintervención por recurrencia de abscesos intraabdominales alcanza el $32 \%$ de los casos (31), razón por la cual en nuestra institución consideramos que el manejo ideal es quirúrgico y solo se deja el tratamiento percutáneo en aquellos casos en los cuales la morbimortalidad del procedimiento quirúrgico es muy alta.

\section{CONCLUSIÓN}

Los cálculos abandonados deben reconocerse como una fuente potencial para la formación de colecciones intraabdominales secundarias a una reacción a un cuerpo extraño que, en ocasiones, puede comprometer otras estructuras como la pared abdominal, el diafragma o extenderse al tórax. Aunque los síntomas son inespecíficos, el dolor abdominal difuso o en el hipocondrio derecho, la distensión abdominal en pacientes con antecedente de CL hace varios meses o incluso años, con presencia de colecciones intraabdominales, especialmente si son perihepáticas, debe despertar la sospecha en el clínico y en el radiólogo de una reacción a un cuerpo extraño por un cálculo abandonado y no debe dejar duda en los casos en los cuales se identifique el cálculo dentro una colección.

Dado el potencial de complicaciones que pueden tener los cálculos libres, es fundamental que el cirujano reconozca una perforación durante la colecistectomía y trate de extraer todos los cálculos libres, y también debe realizar un exhaustivo lavado de la cavidad abdominal. En aquellos pacientes que desarrollaron alguna complicación debido a un cuerpo extraño, definitivamente consideramos que el drenaje percutáneo no ofrece ninguna ventaja, ya que no es un tratamiento definitivo por el alto índice de recurrencia. Por lo tanto, el manejo quirúrgico con extenso lavado de la cavidad es el procedimiento de elección. 


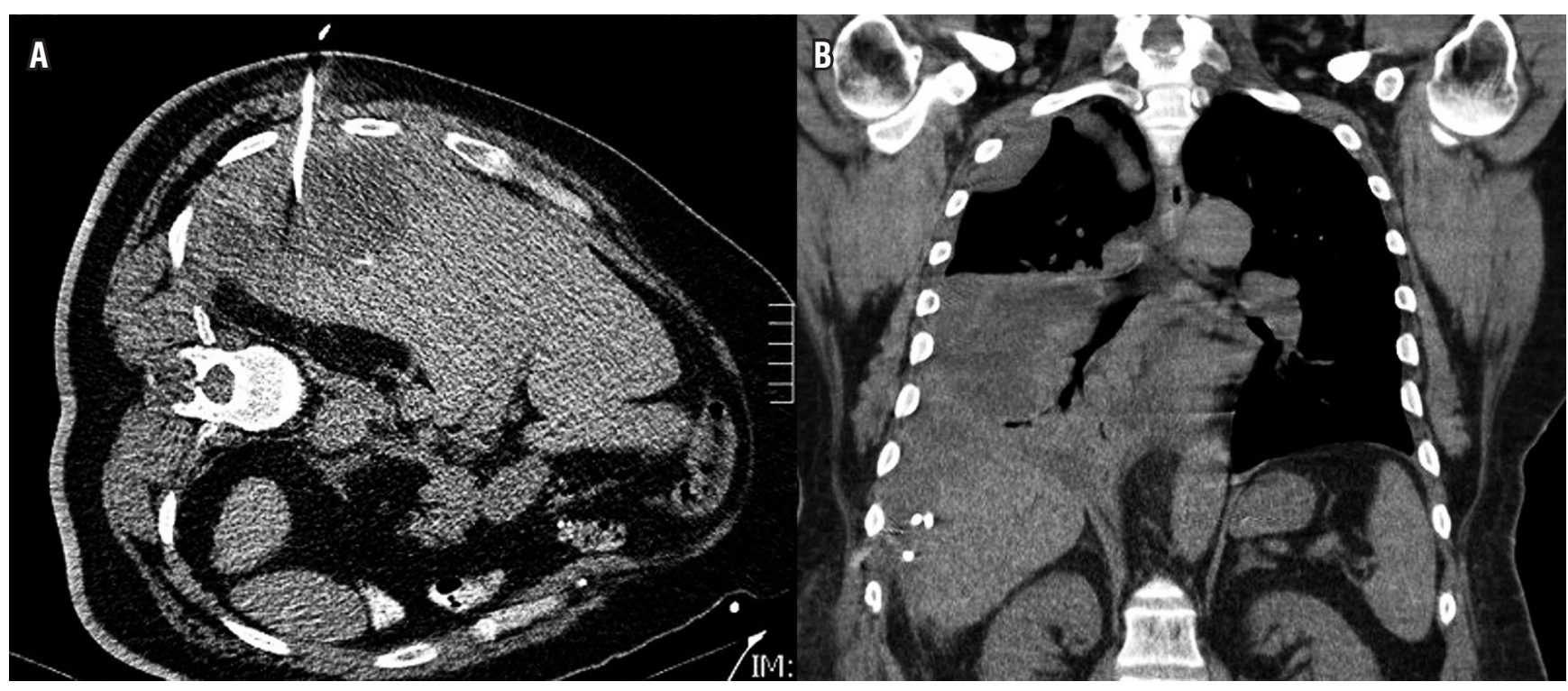

Figura 9. A. Imagen de tomografía computarizada abdominal simple como guía para drenaje percutáneo de colección subhepática derecha. B. Reconstrucción coronal de TC de tórax del mismo paciente, en la que se observa colección pleural tabicada por drenaje a tórax de la colección después de 24 horas del manejo percutáneo. Obsérvese el extremo del catéter pigtail $\left({ }^{*}\right)$.

\section{Financiación y declaración de conflicto de interés}

Ninguno de los autores declara conflictos de interés. No se contó con ninguna fuente de financiación por parte de la industria farmacéutica u otras entidades para la realización de la revisión.

\section{REFERENCIAS}

1. Castellón CJ,FernándezM,Morales S, etal.Absceso subhepático como complicación tardía de un cálculo intraperitoneal abandonado tras una colecistectomía laparoscópica. Gastroenterol Hepatol. 2004;27(10):568-72. Doi: https://doi.org/10.1157/13069130 / https://doi. org/10.1016/S0210-5705(03)70534-2

2. Atri M, Bonifacio A, Ryan M, et al. Dropped gallstones post laparoscopic cholecystectomy mimicking peritoneal seeding: CT and ultrasound features. J Comput Assist Tomogr. 2002;26(6):1000-5. Doi: https://doi. org/10.1097/00004728-200211000-00025

3. Morrin MM, Kruskal JB, Hochman MG, et al. Radiologic features of complications arising from dropped gallstones in laparoscopic cholecystectomy patients. AJR Am J Roentgenol. 2000;174(5):1441-5. Doi: https://doi. org/10.2214/ajr.174.5.1741441

4. Zehetner J, Shamiyeh A, Wayand W. Lost gallstones in laparoscopic cholecystectomy: all possible complications. Am J Surg. 2007;193(1):73-8. Doi: https://doi.org/10.1016/j. amjsurg.2006.05.015
5. Werber YB, Wright CD. Massive hemoptysis from a lung abscess due to retained gallstones. Ann Thorac Surg. 2001;72:27880. Doi:https://doi.org/10.1016/S0003-4975(00)02563-7

6. Singh AK, Levenson RB, Gervais DA, et al. Dropped gallstones and surgical clips after cholecystectomy: CT assessment. J Comput Assist Tomogr. 2007;31(5):758-62. Doi: https:// doi.org/10.1097/RCT.0b013e3180340358

7. ZaliekasJ,MunsonJL. Complications ofgallstones: the Mirizzi syndrome, gallstone ileus, gallstone pancreatitis, complications of "lost" gallstones. Clin North Am. 2008;88(6):134568. Doi: https://doi.org/10.1016/j.suc.2008.07.011

8. Suyapto D, Tian-Hui J. Complications of retained intraperitoneal gallstones from laparoscopic cholecystectomy. Surg Laparosc Endosc Percutan Tech. 2006;16(3):167-8. Doi: https://doi.org/10.1097/00129689-200606000-00010

9. Khan M, Khatri M, Oonwala Z. Knowledge and practices of general surgeons and residents regarding spilled gallstones lost during laparoscopic cholecystectomy: a cross sectional survey. Patient Safety in Surgery. 2013;7:27. Doi: https:// doi.org/10.1186/1754-9493-7-27

10. Eikermann M,SiegelR,BroedersI, etal.Prevention and treatment of bile duct injuries during laparoscopic cholecystectomy: the clinical practice guidelines of The European Association for Endoscopic Surgery (EAES). Surg Endosc. 2012;26(11):300339. Doi: https://doi.org/10.1007/s00464-012-2511-1

11. Randial Pérez LJ, Fernando Parra J, Aldana Dimas G. The safety of early laparoscopic cholecystectomy ( $<48$ hours) for patients with mild gallstone pancreatitis: a systematic review of the literature and meta-analysis. Cir Esp. 2013. Doi: https://doi.org/10.1016/j.ciresp.2013.01.024 
12. Whiting J, Welch Nt, Hallissey MT. Subphrenic abscess caused by gallstones "lost" at laparoscopic cholecystectomy one year previously: Management by minimally invasive techniques. Surg Laparosc Endosc. 1997;7(1):77-8. Doi: https://doi.org/10.1097/00019509-199702000-00022

13. Fitzgibbons RJ, Annibali R, Litke BS. Gallbladder perforation and gallstone removal: open versus closed laparoscopy and pneumoperitoneum. Am J Surg. 1993;165:497-504. Doi: https://doi.org/10.1016/S0002-9610(05)80949-3

14. Karabulut N, Tavasli B, Kiroğlu Y. Intra-abdominal spilled gallstones simulating peritoneal metastasis: CT and MR imaging features (2008: 1b). Eur Radiol. 2008;18(4):851-4. Doi: https://doi.org/10.1007/s00330-007-0703-1

15. Habib E, Elhadad A. Digestive complications of gallstones lost during laparoscopic cholecystectomy. HPB (Oxford). 2003;5(2):118-22. Doi: 10.1080/13651820310016463.

16. Gayer G, Petrovitch I, Jeffrey RB. Foreign objects encountered in the abdominal cavity at CT. Radiographics. 2011;31(2):40928. Doi: https://doi.org/10.1148/rg.312105123

17. Dida K, Mostafa G. Complications of spilled gallstones after laparoscopic cholecystectomy. Am Surg. 2013;79(3):E106-7.

18. Brockmann JG, Kocher T, Senninger NJ, et al. Complications due to gallstones lost during laparoscopic cholecystectomy. Surg Endosc. 2002;16(8):1226-32. Doi: https://doi. org/10.1007/s00464-001-9173-8

19. Van Mierlo PJ, De Boer SY, Van Dissel JT, et al. Recurrent staphylococcal bacteraemia and subhepatic abscess associated with gallstones spilled during laparoscopic cholecystectomy two years earlier. Neth J Med. 2002;60(4):177-80.

20. Memon MA, Deeik RK, Maffi TR, et al. The outcome of unretrieved gallstones in the peritoneal cavity during laparoscopic cholecystectomy. Surg Endosc. 1999;13:848-57. Doi: https://doi.org/10.1007/s004649901118

21. Schäfer M, Suter C, Klaiber $\mathrm{Ch}$, et al. Spilled gallstones after laparoscopic cholecystectomy. A relevant problem? A retrospective analysis of 10,174 laparoscopic cholecystectomies. Surg Endosc. 1998;12:305-9. Doi: https://doi. org/10.1007/s004649900659
22. Horton M, Florence MG. Unusual abscess patterns following dropped gallstones during laparoscopic cholecystectomy. Am J Surg. 1998;175:375-9. Doi: https://doi.org/10.1016/ S0002-9610(98)00048-8

23. Iannitti DA, Varker KA, Zaydfudim V, et al. Subphrenic and pleural abscess due to spilled gallstones. JSLS. 2006;10(1):101-4.

24. Hussain S. Sepsis from dropped clips at laparoscopic cholecystectomy. Eur J Radiol. 2001;40(3):244-7. Doi: https:// doi.org/10.1016/S0720-048X(00)00163-7

25. Nayak L, Menias CO, Gayer G. Dropped gallstones: spectrum of imaging findings, complications and diagnostic pitfalls. Br J Radiol. 2013;86(1028):20120588. Doi: https:// doi.org/10.1259/bjr.20120588

26. Helme S, Samdani T, Sinha P. Complications of spilled gallstones following laparoscopic cholecystectomy: a case report and literature overview. J Med Case Rep. 2009;3:8626. Doi: https://doi.org/10.4076/1752-1947-3-8626

27. Birkitt DH. Spilled cells, spilled clips, spilled stones. Editorial. Surg Endosc. 1995;9:269-71. Doi: https://doi. org/10.1007/BF00187766

28. Soper NJ, Dunnegan DL. Does intraoperative gallbladder perforation influence the early outcome of laparoscopic cholecystectomy? Surg Laparosc Endosc. 1991;1:156-61.

29. Tumer A, Yüksek Y, Yasti A, et al. Dropped gallstones during laparoscopic cholecystectomy: the consequences. World J Surg. 2005;29:437-40. Doi: https://doi.org/10.1007/ s00268-004-7588-9

30. Rice DC, Memon MA, Jamison RL, et al. Long-term consequences of intraoperative spillage of bile and gallstones during laparoscopic cholecystectomy.J Gastrointest Surg. 1997;1:8591. Doi: https://doi.org/10.1007/s11605-006-0014-x

31. Castellón-Pavón C, Morales-Artero S, Martínez-Pozuelo A, et al. Complicaciones por cálculos y clips intraabdominales abandonados durante una colecistectomía laparoscópica. Cir Esp. 2008;84(1):3-9. Doi: https://doi.org/10.1016/ S0009-739X (08)70596-7 